\title{
An atypical presentation of swelling in the floor of the mouth - A case report
}

\section{Nerella Narendra Kumar ${ }^{1, *}$, Savitha S Shettar ${ }^{2}$, Mamatha Gowda Panchaksharappa ${ }^{3}$, Rajeshwari G Annigeri ${ }^{4}$}

${ }^{1}$ Senior Lecturer, ${ }^{2}$ Government Dental Health Officer, ${ }^{3}$ Professor, ${ }^{4}$ Professor and HOD, ${ }^{1}$ Narayana Dental College and Hospital, Nellore, Andhra Pradesh, ${ }^{2}$ Community Health Centre, Wadagera, Yadgiri, Karnataka, ${ }^{3,4}$ College of Dental Sciences, Davanagere, Karnataka, India

\section{*Corresponding Author: Nerella Narendra Kumar}

Email: nerellanarendra@gmail.com

\begin{abstract}
Adenoid cystic carcinoma (ACC) is a neoplasm arising from salivary gland epithelium that affects commonly minor salivary glands, among which palate being the most common site. ACC accounts for about $1 \%$ of malignancies that do affect the head and neck region. It usually affects the individuals in the $5^{\text {th }}$ and $6^{\text {th }}$ decade of life. Clinical course is characterized by indolent, locally invasive growth with high propensity for perineural invasion, local recurrence and distant metastasis. Here we report a case with the unusual presentation of $\mathrm{ACC}$ in the young male patient.
\end{abstract}

Keywords: Swelling, Floor of the mouth, Salivary gland, Adenoid cystic carcinoma.

\section{Introduction}

Salivary gland neoplasms may present in a diversified manner of their distinct histological characteristics and clinical behavior. This marked variation warrants the thorough knowledge of anatomy and physiology, as well as expertise in pathology. ${ }^{1}$

Adenoid cystic carcinoma (ACC) is commonest of all the salivary gland lesions. It accounts for $1 \%$ of all malignancies that occur in oral and maxillofacial region. $22 \%$ of all salivary gland malignancies were ACC. ${ }^{2}$

ACC frequently affects minor salivary glands; palate being the most common site. It poses a problem at the time of diagnosis often recognized at the advanced stage and do possess a problem for complete resection being larger in size and its proximity to important neural and vascular structures. $^{3}$

This case report describes a unique case of ACC in the floor of the mouth with atypical presentation in a young male patient.

\section{Case Report}

A 26-year-old male patient reported to the Department of Oral Medicine and Radiology with the chief complaint of pain in the lower right back tooth region since 5 days and swelling below the tongue since 2 days. Pain was sudden in onset, intermittent, moderate in intensity, non - radiating type, aggravated by taking foods and relieved on its own. Patient noticed the swelling below the tongue since 2 days. The size of the swelling remains same since its occurrence. It was not associated with the pain. No history of ulceration or bleeding/pus discharge. No history of alteration in saliva quantity and quality, taste alteration and not associated with any difficulty in mastication, swallowing $\&$ articulation. No history of fever, loss of weight, trauma, recurrence or any altered sensation is evidenced No history of similar swelling elsewhere in the body and no associated habits are noticed.

The patient's past dental and medical histories were non-contributory to this complaint. No abnormality detected on extra- oral examination. Intraorally a smooth pink colored ovoid swelling of approximately $2.1 \times 2.3 \mathrm{cms}$ present in the right side of the floor of the mouth extending from the distal surface of 46 to the mesial surface of the 41 and from the lingual side of the alveolar ridge crossing the midline upto distal surface of 32 .No visible pulsations and no visible bleeding/ discharge present. Salivary duct orifices appear obliterated. On bidigital palpation it was non-tender, hard in consistency and freely mobile in antero-posterior direction without any signs of bleeding/discharge, ulceration, induration, paraesthesia or anaesthesia. Salivary flow was normal from the ductal openings. (Fig. 1)

Hard tissue examination revealed a root stump i.r.t 46 with obliteration of lingual vestibule. Other findings were root stumps i.r.t.16, 26; grossly decayed teeth i.r.t.48, dental caries i.r.t 15, 24, 28 with mild amount of calculus and stains. Based on a detailed history, careful clinical examination, provisional diagnosis of chronic periapical abscess of 46 with the differential diagnosis of sialolith in the submandibular duct, minor salivary gland tumor was considered.

Mandibular true occlusal radiograph revealed no abnormality and panoramic radiograph revealed a solitary radiolucency in the periapical region of 46 (Fig. 2). Ultrasonography revealed a well defined hypoechoic solid lesion seen on the right side of the frenulum in the floor of the mouth about $2.7 \times 2.3 \mathrm{cms}$ with minimal vascularity (Fig. 3 ). FNAC revealed a few clumps as well as isolated cells of salivary gland with very scarce amount of inflammatory cells. Cells are clumped by fibrillary stroma and focally placed mucous globules which are suggestive of benign salivary gland tumor.

Considering the benign nature of the lesion, lesion was excised along with the extraction of 46. Excisional biopsy revealed a salivary gland tissue with an encapsulated tumor with the cells arranged in a cribriform pattern along with the tumor vascular emboli. Focal areas of tumorcell infiltration in the inner half of the capsule were suggestive of adenoid cystic carcinoma (Fig. 4). Further total body scan was performed which was negative for distant metastasis. Based on the clinical features and the histopathologic report, a final diagnosis of Adenoid cystic carcinoma of minor 
salivary glands of floor of the mouth T2 N0 M0 \& Grade-I was made. Following the post-operative period of 3 years

the patient was disease free.

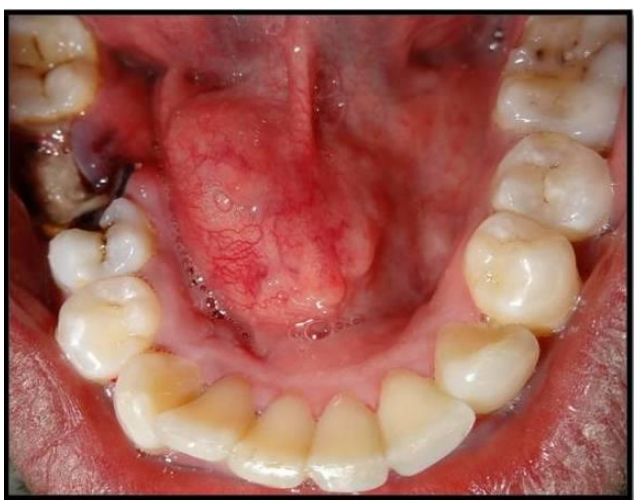

Fig. 1: Clinical Presentation of a Swelling

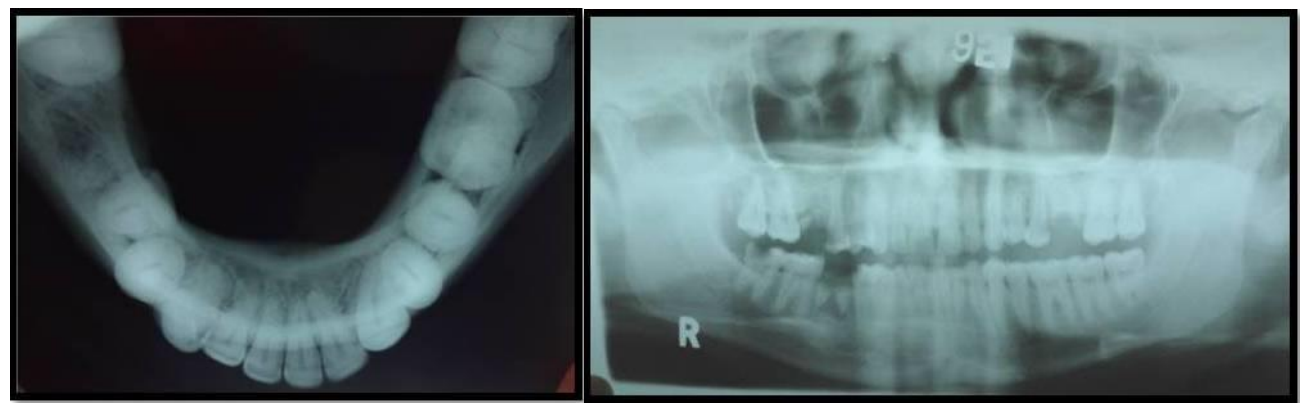

Fig. 2: Occlusal and panoromic images

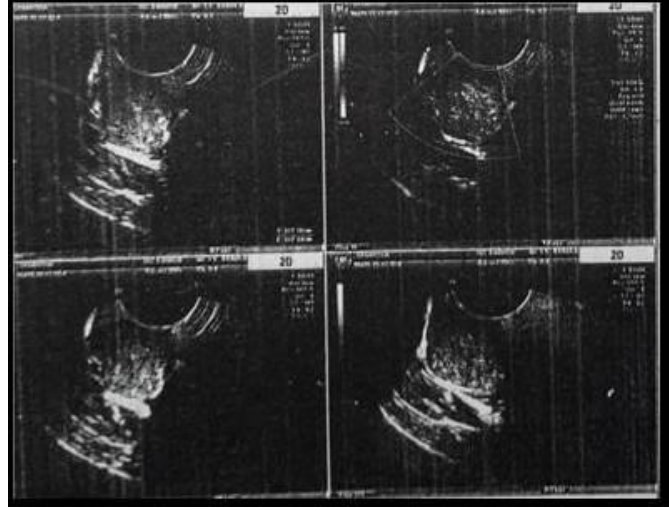

Fig. 3: Ultrasonographic images of the swelling

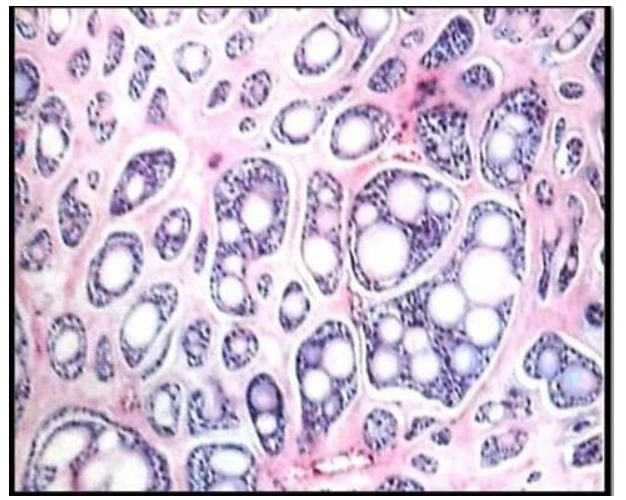

Fig. 4: 20X microphotograph showing cribriform pattern

\section{Discussion}

Three Frenchmen- Robin, Lorain, and Laboulben first described ACC. Billroth, in1859, described it as cylindroma based on the cylindrical nature of its appearance. Spies in 1930, coined the term adenoid cystic carcinoma. ${ }^{2,3}$

ACC is defined by the World Health Organization as "a basaloid tumour consisting of epithelial and myoepithelial cells in various morphological configurations, including tubular, cribriform and solid patterns. It has a relentless clinical course and usually a fatal outcome". ${ }^{4}$

ACC can appear at virtually any age, common being the $5^{\text {th }}$ or $6^{\text {th }}$ decade of life. Gender predilection is an inconsistent feature in the literature; however, it is slightly more common in women than in men. ${ }^{5}$ In contrast to this; our case was reported in 26 year old male patient who was very unusual at the younger age and the gender wise.

Its predilection for the site of occurrence is minor salivary glands of oral cavity, the major salivary glands, and the extraoral seromucinous glands. ${ }^{2}$ Palate accounts for almost $60 \%$ of all ACCs that do affect the minor salivary glands, followed by the floor of the mouth, the tongue and the gingiva. ${ }^{3,4}$ In the present case, the floor of the mouth was the site.

ACC is usually asymptomatic in nature and slow growing in nature. In majority of the cases, it becomes symptomatic until and unless when it invades the local nerves and related structures. Symptoms depend upon the site of invasion and patient presents with a locally invaded 
disease. It has a high rate of recurring tendency and metastases to various organs, lung being the commonest one. Pain is the commonest reported symptom, before the clinical evidence of the lesion as its propensity towards the neurological invasion. ${ }^{6}$ Two days swelling was the reported complaint without any pain in the present case suggestive of non -invading nature. Even the distant metastasis is not evident in our case which is suggestive of an early lesion. When we are considering such lesions of shorter duration, these entities have to be kept in mind when considering the differential diagnosis.

The fine needle aspiration biopsy of ACC characteristically showed small clusters or groups of basaloid tumor cells encircling the hyaline globule. Tumor cells contain small, ovoid nucleus with mild to moderate pleomorphism and hyperchromasia. ${ }^{7}$ In our case, FNAC is suggestive of benign salivary gland tumor which doesn't show any features of ACC.

Histologically, ACC presents in three different patterns: cribriform, tubular and solid. Tumor is graded according to Szanto et al., cribriform or tubular (grade I), less than $30 \%$ solid (grade II), or greater than $30 \%$ solid (grade III). The most important and classical feature of it is the "cribriform" pattern where nests of tumor cells have a sievelike or "Swiss cheese" configuration. A second major pattern observed in ACC is the "tubular" pattern in which elongated tubular structures with a central lumen are seen. The third pattern is the "solid" pattern where the tumor nests and islands are completely filled with basaloid tumor cells without cystic spaces. $^{8}$

Prognosis of ACC depends on the site, size and extension into adjacent structures. ${ }^{9}$ Mortality rate is in consistent with the perineural invasion and degree of mitoses. ${ }^{10}$ various treatment modalities are surgical excision, radiotherapy, chemotherapy, and combined therapy. Site, stage, biological behavior and histological nature of ACC dictate the choice of the therapy. Radical surgery combined with radiotherapy is the commonest one. Post-operative radiotherapy is indicated for tumors associated with perineural spread, local recurrence, and distant metastases. ${ }^{3}$

In the present case, first we thought of simple entity periapical abscess, then we started with a course of antibiotics and analgesics and when the patient is followed up for five days only the pain associated with the teeth was subsided and swelling remains same. Then further investigations were carried out which resulted in a benign salivary gland tumor, so surgical treatment was planned along with the extraction of the root stump. Excisional biopsy turned out into ACC and further body scan was done to rule out metastasis which was negative. In spite of only surgical management, 3 years follow-up showed no recurrence. This may be due to better prognosis associated with the less aggressive cribriform type of the lesion. Further studies are required to validate treatment with only surgery in cases of tubular and cribriform forms of ACCs.

\section{Conclusion}

Patient comes with some simple complaint. If not examined and followed up properly, he can be landed into a different one which can be a life taking. The present case highlights the need to be aware of unusual presentation of ACC which seems to be a simple swelling but warrants the proper examination and follow-up.

\section{Conflict of Interest: Nil.}

\section{References}

1. Tincani AJ, Del Negro A, Araújo PP, Akashi HK, Martins AS, Altemani AM, Barreto G. Management of salivary gland adenoid cystic carcinoma: institutional experience of a case series. Sao Paulo Med J. 2006;124(1):26-30.

2. Jaso J, Malhotra R. Adenoid cystic carcinoma. Arch Pathol Lab Med. 2011;135(4):511-515.

3. Bradley PJ. Adenoid cystic carcinoma of the head and neck: a review. Curr Opin Otolaryngol Head Neck Surg. 2004;12(2):127-132.

4. El-Naggar AK, Huvos AG. Adenoid cystic carcinoma. In: Barnes L, Eveson JW, Reichart P, Sidransky D, eds. Pathology and genetics of head and neck tumors. World Health Organization classification of tumors. Lyon: IARC Press; 2005.

5. Khan S, Agwani K, Bhargava P, Kumar SP. Adenoid cystic carcinoma presenting as an ulcer on the floor of the mouth: a rare case report. J Korean Assoc Oral Maxillofac Surg. 2014;40(5):253-257.

6. Pushpanjali M, Sujata DN, Subramanyam SB, Jyothsna M. Adenoid cystic carcinoma: An unusual presentation. J Oral Maxillofac Pathol. 2014;18:286-290.

7. Dutta NN, Baruah R, Das L. Adenoid cystic carcinoma-Clinical presentation and cytological diagnosis. Indian J Otolaryngol Head Neck Surg. 2002;54:62-4.

8. Szanto PA, Luna MA, Tortoledo ME. Histologic grading of adenoid cystic carcinoma of the salivary glands. Cancer. 1984;54:1062-1069.

9. Spiro RH, Huvos AG, Strong EW. Adenoid cystic carcinoma of salivary origin. A clinicopathologic study of 242 cases. Am J Surg. 1974;128:512-520.

10. Blanck C, Eneroth CM, Jacobsson F, Jakobsson PA. Adenoid cystic carcinoma of the parotid gland. Acta Radiol Ther Phys Biol. 1967;6:177-196.

How to cite this article: Kumar N. N, Shettar S. S, Panchaksharappa M. G, Annigeri R. G. An atypical presentation of swelling in the floor of the mouth - A case report. Int J Oral Health Dent. 2018;4(4):239241. 